\title{
Electrochemiluminescence Detection of Methamphetamine in Biological Matrices
}

\author{
E. Dokuzparmak, L Dennany* \\ ${ }^{a}$ WESTChem, Department of Pure and Applied Chemistry, University of Strathclyde, Technology and \\ Innovation Centre, 99 George Street, Glasgow, G1 1RD, UK \\ *lynn.dennany@strath.ac.uk; phone+44141548 4322
}

\begin{abstract}
We have studied an electrochemical detection method for the stimulants in the forensic samples using electrochemiluminescence (ECL). In this context, amphetamine type stimulant (methamphetamine (MA)) has been studied as coreactants in the $\left[\mathrm{Ru}(\mathrm{bpy})_{3}\right]^{2+}$ (where bpy is $2,2^{\prime}$-bipyridine) ECL system. This approach is developed based on a glassy carbon electrode modified with $\left[\mathrm{Ru}(\mathrm{bpy})_{3}\right]^{2+} / \mathrm{Nafion}$ composite film. LoD, LoQ and linear working range for MA are studied currently. The ECL intensity was found to be concentrations over the range of $5 \times 10^{-8}$ to $2.5 \times 10^{-4} \mathrm{~mol} / \mathrm{L}$. LoD for MA is $1.94 \times 10^{-10}$ $\mathrm{mol} / \mathrm{L}$. The regression coefficient is 0.9931 for the experiment. Our approach was applied in different medium such as saliva and human serum to detect MA This technique is simple, rapid, selective and sensitive, and shows potential for the highthroughput quantitation of MA. the results show that the present electrochemical approach seems to provide a sensitive detection of MA in forensic applications.
\end{abstract}

Keywords: Electrochemiluminescence, biological matrices, drug detection, toxicological detection

\section{INTRODUCTION}

Amphetamine type substances (ATS) continue to be abused globally ${ }^{1}$. Indeed around 27 million people worldwide are estimated to have used ATS, with the use of methamphetamine (MA) increasing in many countries including the US, Australia and the UK to name a few. ${ }^{1}$ Other ATS include methylenedioxyamphetamine, and methylenedioxyethyle-amphetamine (MDEA) as highlighted in Figure 1. MA is a potent central nervous system stimulant and is mainly used for recreational drug. There are two enantiomers of MA and they are levo-methamphetamine and dextro-methamphetamine. In pure amine forms, these enantiomers are equal in the mix and show racemic property. Dextro-methamphetamine is a much stronger central nervous system than levo-methamphetamine. ${ }^{2}$ Dextro-methamphetamine is generally in the form of a hydrochloride salt and this salt is called as 'ice' and 'crystal math'. The crystal math allows users to inhale the vapor of MA. This ability of hydrochloric form of MA enables higher and faster recreational effect. ${ }^{2}$

This is a peer reviewed, accepted author manuscript of the following research article: Dokuzparmak, E., \& Dennany, L. (2020). Electrochemiluminescence detection of methamphetamine in biological matrices. Proceedings of SPIE, 11542, [115420D]. https://doi.org/10.1117/12.2573548 
The determination of ATS is important within medicine fields due to the toxicological and addictive effects of ATS. The standard approach for determining the abuse and / or use of MA is usually completed by verifying the concentration of MA in biological samples such as urine. This is most often completed using immunoassays or chromatographic techniques which both require sample preparation before analysis. ${ }^{2,3}$ Immunoassays, in particular, are often the primary approach for the screening of illicit substances. ${ }^{3,4}$ However, specificity can be a challenge especially for determining which ATS is present. ${ }^{3,5}$ The need for rapid detection and monitoring in clinical, environmental and forensic fields has seen increased research into this area with an emphasis on low-cost and reusable analytical devices. Electrochemical biosensors, including electrochemiluminescence (ECL) based sensors, have a wide range application in food, pathogens, forensic applications, drug response measurement, cancer diagnosis and environmental issues. ${ }^{6,7,8}$

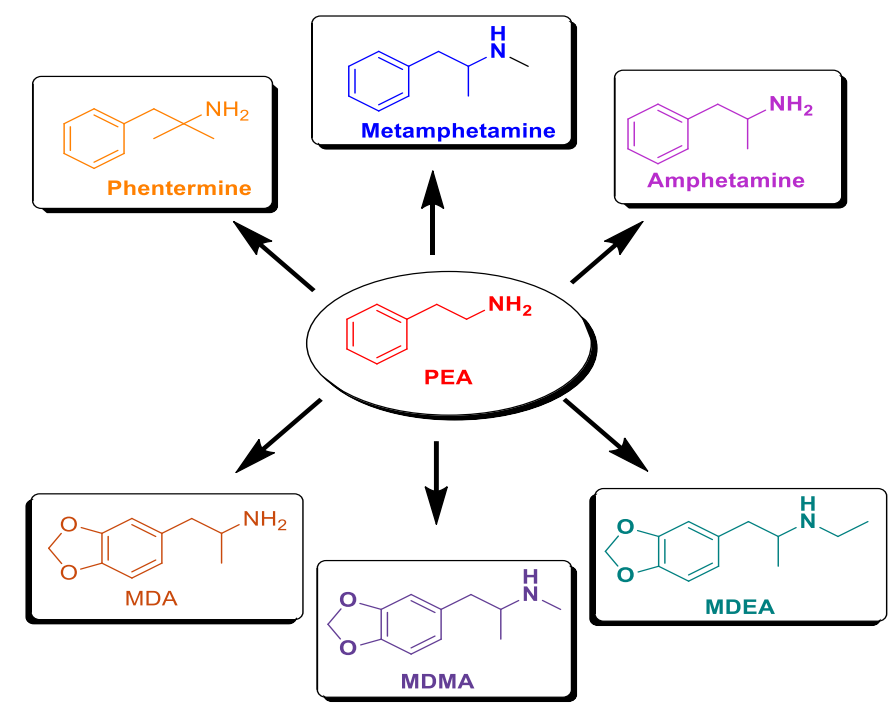

Figure 1. Molecular structures of amphetamine, methamphetamine, 3,4-methylenedioxy-amphetmaine (MDA), methylenedioxyethyleamphetamine (MDEA) and 3,4-methylenedioxy-methamphetamine (MDMA).

Electrochemical sensors, including electrochemiluminescence (ECL) can offer a viable alternative for applications within the forensic and clinical environments. The expansion of current developments within the field has seen the introduction of portable sensors which can be controlled via commercially available handheld devices or smartphone devices. ${ }^{8,9,10,11,12,13,14}$ One of the major advantages of ECL is the greater control over both the position and time of ECL reactions. ECL emission occurs only in the immediate vicinity of the electrode, which is beneficial for selectivity, multi-analyte detection using multiple electrodes, and sensitivity. Control over the timings of the reaction improves reproducibility. ${ }^{15}$ These advantages have resulted in the application of ECL across many diverse research fields, including food and water testing, clinical diagnostics and bio-warfare agent detection. ${ }^{16,17}$ There is also an increasing interest in oral fluid as alternative to plasma sample or urine in clinical and 
forensic cases because oral fluid can be collected directly and there is no need for an additional process when direct observation. ${ }^{4}$ Based on the results in the literature, the samples of oral fluid show similar results to those of plasma and drug concentrations in oral fluid reflect concurrent plasma concentrations. ${ }^{4,6}$

Saliva is a mixture of secretions from three major and minor accessory salivary glands and commonly is referred to as saliva. Bacteria and food debris are also present, but the main and major component is water ${ }^{7}$. Oral fluid is hypotonic compared to plasma and there is a considerable variety in ionic concentrations of resting and stimulated states. $\mathrm{pH}$ of oral fluid in healthy individuals is usually between 6.2 and 7.4. An adult may produce $\sim 1000 \mathrm{~mL} /$ day of oral fluid with typical flows of 0.05 $\mathrm{mL} / \mathrm{min}^{5}{ }^{5}$ The basicity of ATS, with pKa values of $\sim 9.9$ for MA ${ }^{18}$, facilitates the opportunity for detection within oral fluid as they are easily transferred to the saliva. The typical time frame for the detection of MA in saliva is $\sim 1$ hour following the ingestion of a $10 \mathrm{mg}$ does, highlighting the need for a rapid and portable detection approach. The concentrations observed are usually in the $\mathrm{nM}$ range in comparison to the plasma where typically $\mu \mathrm{M}$ concentrations are observed.

With ECL based sensors growing in popularity as alternatives for employment as point-of-care or at-scene devices alongside their proven ability to detect MA, further investigation into their viability for detection of MA in biological matrices is required. Within this contribution, we report on the development of a simple $\left[\mathrm{Ru}(\mathrm{bpy})_{3}\right]^{2+}$ ECL sensor for the rapid screening of MA within biological fluids including human serum and artificial saliva. This will be achieved with minimal sample preparation, providing a screening system capable of offering rapid results required by law enforcement and / or physicians without access to a dedicated laboratory facility.

\section{EXPERIMENTAL}

\subsection{Materials and Reagents}

Tris (2,2'- bipyridyl) - dichlororuthenium (II) hexahydrate, $\left(\left[\mathrm{Ru}(\mathrm{bpy})_{3}\right]^{2+}\right),(+)$ methamphetamine hydrochloride, phosphate buffered saline (PBS) tablets, human pooled serum, and 117 Nafion ( $\sim 5 \%(\mathrm{w} / \mathrm{v})$ mixture of lower aliphatic alcohols and water) were purchased from Sigma-Aldrich. 0.1 M PBS was used as the electrolyte. All other reagents were of analytical reagent grade and ultra-pure water (Milli-Q plus, Millipore Inc.) was used throughout the experiments. Artificial saliva (Bioténe ${ }^{\circledR}$ oral balance gel) and synthetic skin were commercially purchased and used as received.

\subsection{Materials and Reagents}

All electrochemical analysis was performed on a $\mathrm{CH}$ instrument (model 602E) using a standard three-electrode configuration. A glassy carbon electrode (GCE) was coated with $\left[\mathrm{Ru}(\mathrm{bpy})_{3}\right]^{2+} /$ Nafion film as working electrode. A platinum wire electrode and $\mathrm{Ag} / \mathrm{AgCl}$ (saturated $\mathrm{KCl}$ ) were used as counter electrode and reference electrode, respectively. All the potentials were 
measured and reported according to this reference electrode. The ECL experiments were carried using the $\mathrm{CH}$ instrument model 602E connected to a Hamamatsu H6780-20 PMT. The ECL cell was placed directly in front of the photomultiplier tube (PMT) which was enclosed in a light-tight box. A full description of the instrumentation employment can be found within our prior publications. ${ }^{17,20}$

\subsection{Fabrication of [Ru(bpy)3]2+ / Nafion ECL Sensor}

The ruthenium film was prepared by drop casting $5 \mu \mathrm{L}$ of a $1: 11 \mathrm{mM}\left[\mathrm{Ru}(\text { bpy })_{3}\right]^{2+}$ in $0.2 \%$ Nafion $117 / \mathrm{MeOH}(1: 3 \mathrm{v} / \mathrm{v})$ solution onto the surface of a GCE (Figure 2). This was then allowed to dry for 2 hours in the dark. The GCE were then stored in the fridge until required for analysis.

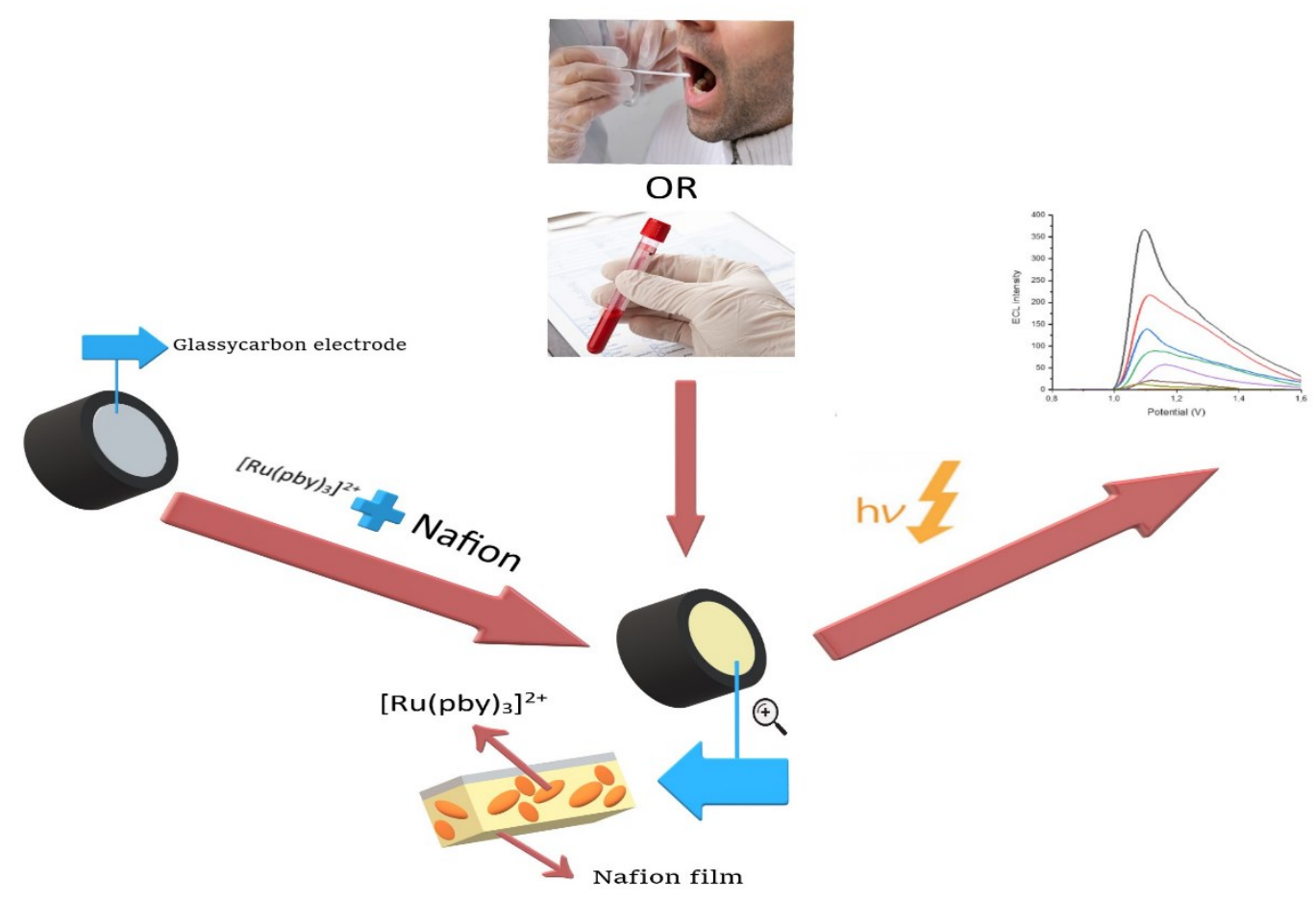

Figure 2. Schematic for the production of this sensor.

\subsection{ECL measurement}

The sensor fabricated was immersed into 0.1 M PBS in the presence of methamphetamine. The ECL measurement was performed at a potential range from $0.0 \mathrm{~V}$ to $1.6 \mathrm{~V}$ at scan rate of 0.1 with a conventional three-electrode system. Glassy carbon electrode fabricated with nafion and $\left[\mathrm{Ru}(\mathrm{bpy})_{3}\right]^{2+}$, a platinum wire and an $\mathrm{Ag} / \mathrm{AgCl}$ (saturated $\mathrm{KCl}$ ) electrode were used as working electrode, counter electrode, and reference electrode, respectively. 


\subsection{Preparation of Biological Samples}

All samples were prepared via spiking of the selected matrix with the required volume of ATS to give the desired concentration. Artificial saliva was commercially purchased. Due to viscosity of the artificial saliva, it was diluted 5 times to observe optimum results. Results from "raw" artificial saliva were also recorded. Human pooled serum samples were stored at $-80{ }^{\circ} \mathrm{C}$ and fully defrosted at room temperature prior to spiking and measurement. Human serum has strong background signal due to some interferences such as amino acids and proteins. To decrease the background signal. Dilution ratio of human serum was investigated, and optimum results was seen at the ratio of 1:3 (Human serum: PBS). Results obtained from non-diluted samples of pooled human serum were also recorded.

\section{RESULTS}

\subsection{Detection of MA via ECL}

Initial investigation of $\left[\mathrm{Ru}(\mathrm{bpy})_{3}\right]^{2+}$ was performed in $0.1 \mathrm{M}$ PBS at $\mathrm{pH} 7.0$ by using glassy carbon electrode. Analysis by $\mathrm{CV}$ was performed at the scan rate range of $100 \mathrm{mVs}^{-1}$ over a potential range of 0.00 to $1.60 \mathrm{~V} \mathrm{vs} \mathrm{Ag} / \mathrm{AgCl}$ (Figure 3).

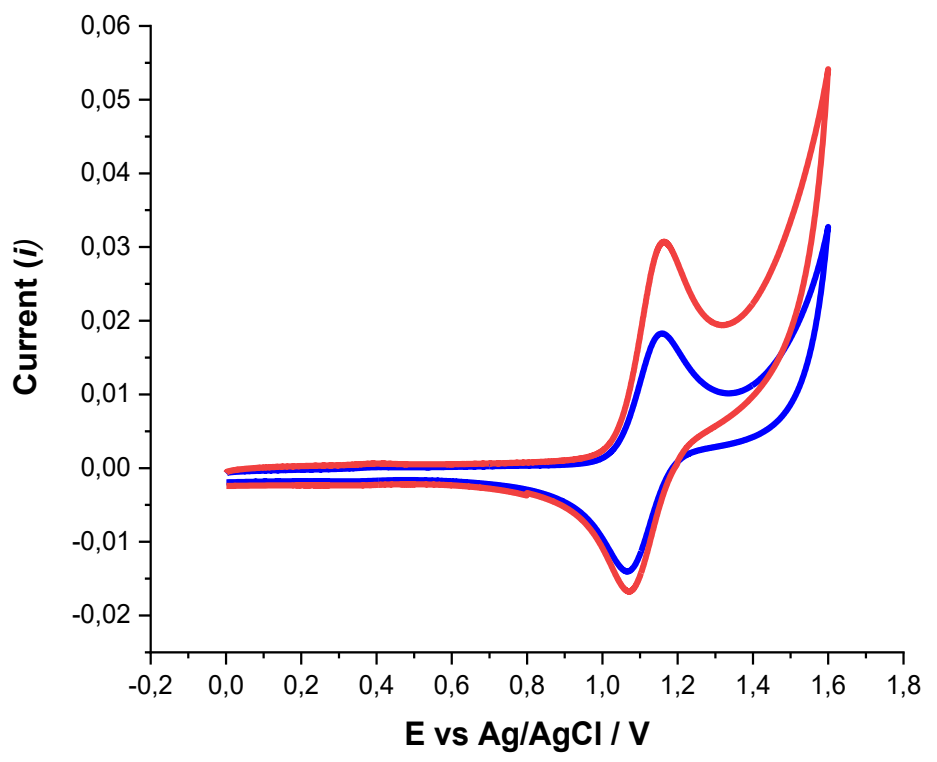

Figure 3. Typical CVs of $\left[\mathrm{Ru}(\mathrm{bpy})_{3}\right]^{2+}$ without MA (blue line) and with MA $(20 \mu \mathrm{M})$ (red line) in $0.1 \mathrm{M}$ PBS at pH 7.0 scanned over the potential range $0.0 \leq E \leq 1.6 \mathrm{~V}$ vs $\mathrm{Ag} / \mathrm{AgCl}$ at a scan rate of $100 \mathrm{mVs}^{-1}$. PMT was biased at $650 \mathrm{~V}$. 
Based on the result from $\mathrm{CVs}$, our system is sensitive to $\left[\mathrm{Ru}(\mathrm{bpy})_{3}\right]^{2+}$ and shows a reversible $\mathrm{CV}$ of $\left[\mathrm{Ru}(\mathrm{bpy})_{3}\right]^{2+}$. When MA is added into the media, an increase in oxidation peak (at about $1.15 \mathrm{~V}$ ) was seen obviously. Our system is sensitive to MA and we can detect the changing in concentration of MA in the medium by $\mathrm{CV}$.

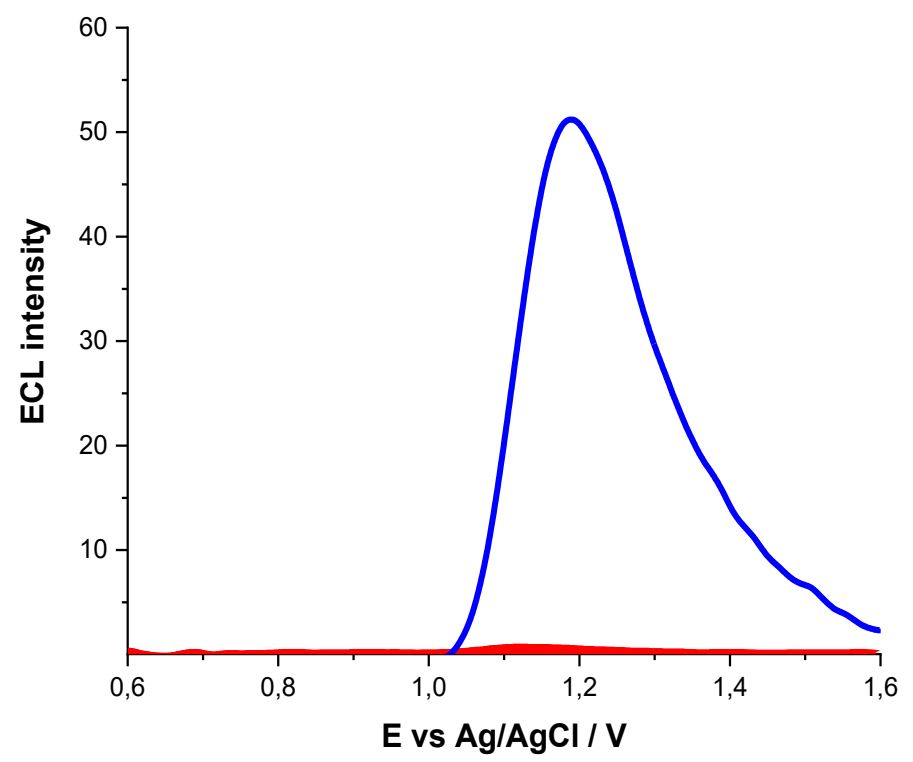

Figure 4. ECL responses for $20 \mu M M A$ in 0.1 M PBS at pH 7.0 (blue line) and blank (red line)

Upon assessing the simultaneous ECL responses, it is clearly evident that the presence of MA has an effect on the ECL behavior of the system which means like the oxalate system. ${ }^{21}\left[\mathrm{Ru}(\mathrm{bpy})_{3}\right]^{2+}$ is mediating the oxidation of methamphetamine which is resulting in the formation of a $\left[\mathrm{Ru}(\mathrm{bpy})_{3}\right]^{\cdot 2+}$ excited state which emits photons of light upon relaxation.

Despite the lack of characterisation of the processes involved in this light emission, it is theorised that methamphetamine follows a similar mechanism to that of TPA co-reactant. The proposed mechanism for the ECL of $\left[\operatorname{Ru}(b p y)_{3}\right]^{2+}$ and methamphetamine is highlighted in Equation 1.1 to 1.4

$$
\begin{aligned}
& {\left[\mathrm{Ru}(\mathrm{bpy})_{3}\right]^{2+}-\mathrm{e}^{-} \longrightarrow\left[\mathrm{MA}^{\cdot}\right]^{+} \underset{\left.\mathrm{Ru}(\mathrm{bpy})_{3}\right]^{3+}}{\longrightarrow} \mathrm{MA}^{\cdot}+\mathrm{H}^{+}} \\
& \mathrm{MA}-\mathrm{e}^{-} \longrightarrow\left[\mathrm{Ru}(\mathrm{bpy})_{3}\right]^{\cdot 2+}+\text { products } \\
& {\left[\mathrm{Ru}(\mathrm{bpy})_{3}\right]^{3+}+\mathrm{MA}^{\cdot} \longrightarrow\left[\mathrm{Ru}(\mathrm{bpy})_{3}\right]^{2+}+\mathrm{h} v_{650 \mathrm{~nm}}}
\end{aligned}
$$




\subsection{Analytical Parameters}

To establish the adequacy of this sensor for both qualitative and semi-qualitative analysis, the analytical parameters were investigated. Previous work has shown that MA can be effectively detected in phosphate buffer ${ }^{9}$ and that the optimal intensity for ECL production is close to the pKa of any amine. However, within this contribution the focus is on the performance at physiological $\mathrm{pH}(\sim \mathrm{pH} 7.0)$ reflecting the $\mathrm{pH}$ for the biological matrices investigated here. As shown in Figure 4, the ECL occurs at a potential of $\sim 1.15 \mathrm{~V}$ vs $\mathrm{Ag} / \mathrm{AgCl}$ and increases with increasing [MA] over the concentration range $10 \mu \mathrm{M}$ to 25 $\mathrm{mM}$. The response is linear over this range (Figure 5) with a LoD of $0.19 \mathrm{nM}$.

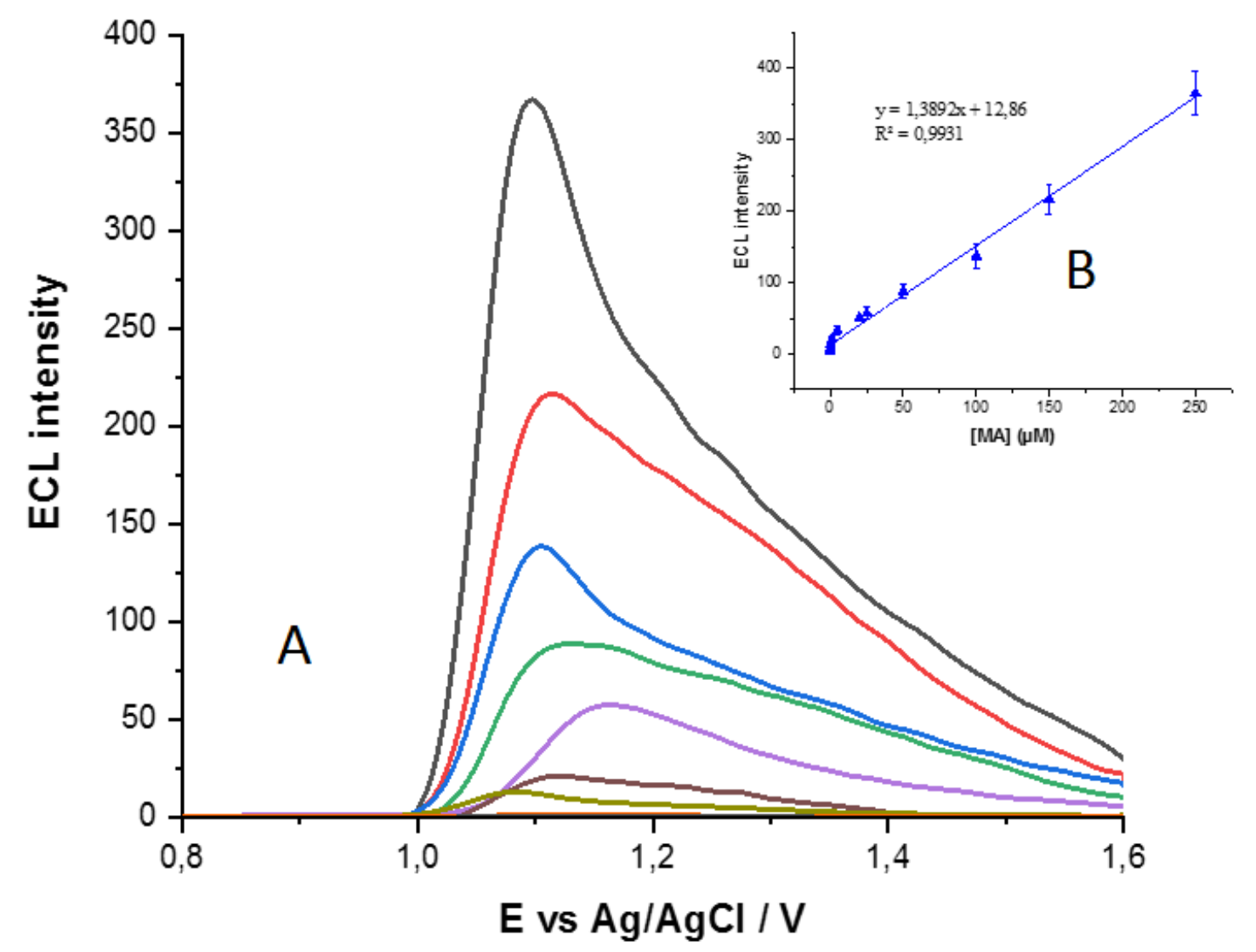

Figure 5. (A) Typical ECL response for increasing [MA] $(0$ to $250 \mu \mathrm{M})$ in $0.1 \mathrm{M}$ PBS at pH 7.0. (B) Limit of detection for MA in $0.1 \mathrm{M}$ PBS at $\mathrm{pH} 7.0$ scanned over the potential range $0.8 \leq E \leq 1.6 \mathrm{~V}$ vs $\mathrm{Ag} / \mathrm{AgCl}$ at a scan rate of $100 \mathrm{mVs}^{-1}$. PMT was biased at $650 \mathrm{~V}$.

This shows a slight improvement on previous ECL based sensors for MA detection. ${ }^{10,13,14}$ It is also possible that the detection limits achieved here could be improved further through the employment of a conducting polymer ${ }^{19}$ rather than nafion to modify the electrode. ECL responses can also be obtained for other $\operatorname{ATS}^{9}$, although these can often produce ECL signals of varying 
intensities and so it should be noted that this approach will not differentiate between the different ATS but can be utilised rather as a screening method for their presence.

Under the condition, ECL intensity of the developed ECL sensor was examined. ECL signals of MA were shown in Figure 5. The ECL intensity is linear with the concentration of MA in the range of $5 \times 10^{-8}$ to $2.5 \times 10^{-4} \mathrm{~mol} / \mathrm{L}$ with the detection of limit of $1.94 \times 10^{-10} \mathrm{~mol} / \mathrm{L}$. The regression coefficient is 0.9931 for the experiment. In the literature ${ }^{10,22,23}$, most ECL sensors have LoD of $10^{-9}$ to $10^{-8}$. Our system has very low detection limit and easy fabrication process. These features can make our system suitable for detection of real samples in forensic cases.

\subsection{Biological fluid analysis}

The biological fluid analysis for human serum and saliva were carried out under the same condition. Due to some amino acids and proteins in serum, high background signal was observed. a dilution step was used to find optimum dilution rate. The serum and saliva were diluted with 0.1 M PBS at $\mathrm{pH}$ 7.0. After the dilution process, three different concentration of MA were examined (100, 150 and $200 \mu \mathrm{M}) . \%$ recovery of saliva and human serum and standart deviations are shown in Tablo 1.

Table $1 . \%$ recovery of MA in diluted saliva and diluted human serum

\begin{tabular}{|c|c|c|}
\hline$[\mathrm{MA}] \boldsymbol{\mu M}$ & $\begin{array}{c}\text { Saliva } \\
\text { \% recovery }\end{array}$ & $\begin{array}{c}\text { Human Serum } \\
\text { \% recovery }\end{array}$ \\
\hline 100 & $97.91 \pm 6.53$ & $103.58 \pm 9.33$ \\
\hline 150 & $98.88 \pm 8.34$ & $101.91 \pm 8.30$ \\
\hline 200 & $100.04 \pm 8.49$ & $95.71 \pm 6.74$ \\
\hline
\end{tabular}

Compared with the results from the literature above, our study has very easy process and easy application and is time saving. There is no need for extraction and pre-treatment of sample. we used a basic dilution just to decrease the background signal. Average of $\%$ recovery of our study in serum as well in saliva was very similar to that of PBS study. \% recovery studies show that our study is suitable for real samples when compared with the results in the literature.

\section{CONCLUSION}

Direct detection of amphetamine type stimulants (ATS) including methylamphetamine (MA) in biological matrices without the need for pre-treatment or extraction is a great challenge for forensic drug analysis. Electrochemical techniques, such as electrochemiluminescence (ECL), are promising tools for this area of analysis. This contribution focuses on the electrochemical and photochemical properties of $\left[\mathrm{Ru}(\mathrm{bpy})_{3}\right]^{2+}$ nafion composite films and their subsequent use for the detection of ATS. The modified films were formed by direct interaction with the electrode surface without the need for surface modification or chain 
linkers. This is a major advantage for the fabrication of any sensor as it reduces the synthesis times resulting in more economically and cheaper production costs. This technique is simple, rapid, selective and sensitive, and shows potential for the high-throughput quantitation of MA.

In conclusion, the present work shows voltammetric and the ECL behaviours of MA in $\left[R u(b p y)_{3}\right]^{2+} /$ nafion film system and this system has high LoD and \% recovery rate in body fluids. The results show that our system can be used for real samples in forensic field. Also, the system can meet the need for fast and cost-effective detection of samples with high sensitivity in forensic field.

\section{ACKNOWLEDGEMENTS}

The authors would like to thank the Ministry of Education of Turkish Republic for financially supporting this research. All data underpinning this publication are openly available from the University of Strathclyde Knowledge Base at https://doi.org/10.15129/eec64b3d-ba2d-4e25-896c-c7b613358792

\section{REFERENCES}

[1] United Nations Publication, World Drug Report 2020, https://wdr.unodc.org/wdr2020/en/drug-use-health.html, (accessed 11th August, 2020).

[2] Moeller, K. E.; Lee, K. C.; Kissack, J. C., "Urine Drug Screening: Practical Guide for Clinicians,” Mayo Clinic Proceedings 83, 66-76 (2008).

[3] Gooch, J., Daniel, B., Parkin, M., \& Frascione, N., "Developing aptasensors for forensic analysis," TrAC Trends in Analytical Chemistry 94, 150-160 (2017).

[4] Graziano, S.; Anzillotti, L.; Mannocchi, G.; Pichini, S.; Busardò, F. P.,"Screening methods for rapid determination of new psychoactive substances (NPS) in conventional and non-conventional biological matrices," J. Pharm. Biomed. Anal 163, 170-179 (2019).

[5] Grafinger, K. E.; Liechti, M. E.; Liakoni, E.,"Clinical value of analytical testing in patients presenting with New Psychoactive Substances intoxication,” Br. J. Clin. Pharmacol. (2019).

[6] Kimmel, D. W., LeBlanc, G., Meschievitz, M. E., \& Cliffel, D. E.,"Electrochemical sensors and biosensors," Analytical chemistry 84(2), 685-707 (2011).

[7] Shaw, L.; Dennany, L., "Forensic drug applications of electrochemical sensors," Curr Opin Electrochem, 3(1), 23-28 (2017).

[8] McGeehan, J.; Dennany, L.,"Electrochemiluminescent detection of methamphetamine and amphetamine," Forensic Sci. Int. 264, 1-6 (2016).

[9] Miao, W.,"Electrogenerated Chemiluminescence and Its Biorelated Applications," Chem. Rev. 108, 2506-2553 (2008).

[10] Richter, M. M.,"Electrochemiluminescence (ECL)," Chem. Rev. 104, 3003-3036 (2004).

[11] Truong, C. K. P.; Nguyen, T. D. D.; Shin, I.S.,"'Electrochemiluminescent Chemosensors for Clinical Applications: A Review," BioChip J. 13, 203-216 (2019).

[12] Gao, W.; Jeanneret, S.; Yuan, D.; Cherubini, T.; Wang, L.; Xie, X.; Bakker, E.,"Electrogenerated Chemiluminescence for Chronopotentiometric Sensors," Anal. Chem. 91, 4889-4895 (2019). 
[13] Doeven, E. H.; Barbante, G. J.; Harsant, A. J.; Donnelly, P. S.; Connell, T. U.; Hogan, C. F.; Francis, P. S.,’"Mobile phonebased electrochemiluminescence sensing exploiting the 'USB On-The-Go' protocol," Sens. Actuators B 216, 608-613 (2015).

[14] Yao, Y.; Li, H.; Wang, D.; Liu, C.; Zhang, C.,"An electrochemiluminescence cloth-based biosensor with smartphonebased imaging for detection of lactate in saliva," Analyst 142, 3715-3724 (2017).

[15]Zheng, H. and Zu, Y.," Emission of Tris(2,2'-bipyridine)ruthenium(II) by Coreactant Electrogenerated Chemiluminescence: From O2-Insensitive to Highly O2-Sensitive," The Journal of Physical Chemistry B 109(24): p. 12049-12053 (2005).

[16] Tombelli, S., Minunni, M., \& Mascini, M.,"Aptamers-based assays for diagnostics, environmental and food analysis," Biomolecular engineering 24(2), 191-200 (2007).

[17] Shan, Y., J Xu, J. and Chen, H.Y.,'Enhanced electrochemiluminescence quenching of CdS:Mn nanocrystals by CdTe QDs-doped silica nanoparticles for ultrasensitive detection of thrombin," Nanoscale 3(7): p. 2916-2923 (2011).

[18] White, R. M.; Moore, C. M., [In Detection of Drugs and Their Metabolites in Oral Fluid], Eds.; Elsevier, pp 11-39 (2018).

[19] Dennany, L. O’Reilly, E.J. Innis, P.C. Wallace, G.G. and Forster R.J.,” The influence of poly (2-methoxyaniline-5-sulfonic acid) on the electrochemical and photochemical properties of a highly luminescent ruthenium complex," Electrochimica Acta 53(13), 4599-4605 (2008).

[21] Lu, M. C., \& Whang, C. W., "The role of direct oxalate oxidation in electrogenerated chemiluminescence of poly (4vinylpyridine)-bound $\mathrm{Ru}$ (bpy) $2 \mathrm{Cl}+$ /oxalate system on indium tin oxide electrodes," Analytica chimica acta 522(1), 2533 (2004).

[22] Sloop, G. Hall, M. Simmons, G. T. Robinson, C. A.,"False-Positive Postmortem EMIT Drugs-of-Abuse Assay due to Lactate Dehydrogenase and Lactate in Urine," J. Anal. Toxicol. 19, 554-556 (1995).

[23] Richter, M. M.," Electrochemiluminescence (ECL)," Chem. Rev. 104, 3003-3036 (2004). 